\title{
Investigation of Key Visual Factors for Cloth Texture Recognition
}

\author{
- Effect of Fabric Drape Complexity and Window Size - \\ Tomoharu ISHIKAWA*, Kou SATO*, Kazuya SASAKI**, \\ Hiroko SHIMIZU** and Miyoshi AYAMA* \\ * Graduate School of Engineering, Utsunomiya University, 7-1-2 Yoto, Utsunomiya, Tochigi 321-8585, Japan \\ ** Faculty of Education, Utsunomiya University, 350 Mine, Utsunomiya, Tochigi 321-8505, Japan.
}

\begin{abstract}
In this paper, we investigate the key visual factors for cloth texture recognition used in the cross-modal linkage between visual and tactile perception. We conducted an experiment to investigate the effects of changes in the complexity of fabric drape shape and the size of the window for observing the fabric on an observer's ability to identify the fabric. The experiment also studied how the estimation of the light-source position affected the accuracy of an observer's identification of a fabric. The experimental results indicated that correct identification of fabric incrementally increases with changes in window size. When the effect of window size is removed, drape complexity is also found to affect accurate fabric identification. In addition, the results indicated that accurately estimating light positions when observing the erected image in the case of high complexity of fabric drape (Comp.15) was directly related to the number of fabrics that were correctly identified.
\end{abstract}

Keywords: Key visual factors, Fabric identification, Complexity of fabric drape shape, Window size,

Accurate estimation of light-source position

\section{INTRODUCTION}

The Internet has become a source for information collection, a means of communication, and an avenue for purchase and consumption; it is becoming a mainstay in people's lives all around the world. Online shopping, by which an individual can purchase a desired object without spending hours in physical stores, has become especially popular among many people regardless of age and gender, and the amount of clothing and accessories purchased online is increasing each year [1]. The buying behavior process for online shopping is called AISCEAS (AttentionInterest-Search-Comparison-Examination-Action-Share). This process includes two features that AIDMA (AttentionInterest-Desire-Memory-Action) — the general buying behavior process - does not include: "Comparison" and "Examination" [1]. For the purpose of online shopping, it is important that the texture of a cloth is faithfully reproduced in a Web image. However, in many cases, the impression of the cloth presented on a Website is different from the tactile feeling of the actual cloth [2], even though high-quality displays are being widely used [3]. This discrepancy is one of the main problems of online shopping. It is necessary to clarify the key visual factors for cloth texture recognition relevant to the tactile perception and to use these factors in the next development of a display. Some trained observers can determine the kind of fabric or quality of cloth quite well even from an image on a low quality display. Understanding how these trained observers identify fabric is useful not only for establishing a better photographic technique that would reduce consumer problems but also for developing an effective educational method for a fashion course. From a scientific point of view, revealing how knowledge and experience affect the cross-modal linkage between vision and touch is also interesting.

In our previous research [4], which investigated the effect of knowledge and experience on one's ability to identify fabric using visual and tactile information, we carried out a fabric-identification experiment using two groups of subjects. One group consisted of engineering students, and the other consisted of clothing students. The two groups had different levels of knowledge and experience, and these factors were measured by a questionnaire. In the fabric-identification experiment, subjects were asked to select a cloth by blind touch based on an image of the cloth on a display. Two kinds of photographs - with and without drapes (Pattern A [PA] and Pattern B [PB]) - were employed as the cloth images. The results indicated that the performance of the clothing students was better than that of the engineering students. The average percent correct was statistically higher for 
students who saw a photograph of PA than for those who saw a photograph of $\mathrm{PB}$ [5]. This result suggests that the existence or nonexistence of a fabric drape was one of the key visual factors for texture recognition. The visual complexity of a fabric drape changes with the setting and lighting method [6], and both pattern perception and visual impressions are affected by the size of the visual field $[7,8]$. Therefore, in this study, we carried out the same fabric-identification experiment, but this time, we focused on three points: the complexity of the fabric drape shape, the size of the window for observing fabric drape, and the accurate estimation of light-source position. The relationship among these three factors and the number of correct answers in the fabric-identification experiment were investigated. Key visual factors for recognizing textures are discussed based on the obtained results.

\section{EXPERIMENT}

\subsection{Experimental Conditions}

\subsubsection{Test Cloths}

Eleven fabrics selected for their different characteristics were prepared for the blind touch experiment. These fabrics are mentioned in Table 1.

Each fabric was cut into a $20 \mathrm{~cm} \times 20 \mathrm{~cm}$ square piece (the size of the conditioned weight at JISL1096 [9]), and a small square tag was attached to the corner of each piece to indicate the top surface of the piece. We called these pieces of fabric "test cloths." An example of a test cloth is shown in Figure 1.

Table 1: Selected 11 fabrics

\begin{tabular}{|l|}
\hline Names of test cloths \\
\hline Silk lace, Ox fabric, Inden-style material, \\
Chiffon crepe, Velour, Velvet, Satin \\
stretch, Satin organdy, Jacquard silk chif- \\
fon, Silk satin, Back satin shantung \\
\hline
\end{tabular}

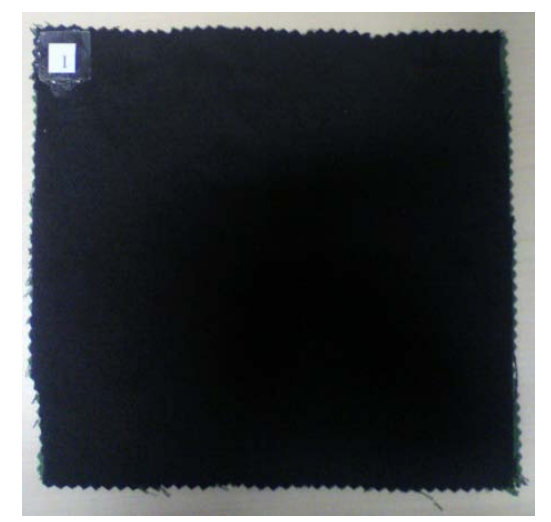

Figure 1: Test cloth (example)

\subsubsection{Test Images and Photography}

Each fabric was also cut into a circular piece, with a diameter of $40 \mathrm{~cm}$, to be photographed. Four photographs were taken of each fabric using a Nikon D50 digital camera with an exposure value of 13.9, a shutter speed of $1 / 80 \mathrm{~s}$, and an aperture of F14. For the first three photographs, the circular cloths were draped over acrylic cylinders of three heights $(1 \mathrm{~cm}, 2 \mathrm{~cm}$, and $15 \mathrm{~cm})$ to make different drape shapes. These photographs were called drape complexity level 1, 2, or 15 depending on the height of the acrylic cylinder. The diameter of the acrylic cylinders was $12 \mathrm{~cm}$, in accordance with drape-testing standards [9]. The fourth photograph was of the circular cloth placed flat on a table. This photograph showed no fabric drape and was called drape complexity level 0 . Figure 2 shows how the photography equipment was set up. The four photographs of Back satin shantung are shown in Figures 3(a)-(d), as an example. Forty-four photographs were taken and used as test images.

\subsubsection{Experimental Stimuli}

To investigate the accurate estimation of light-source positions, 88 test images, divided into 2 groups of 44, were made. One group consisted of the original 44 test images, called the erected images. Another group consisted of the images processed by inverting the original photographs; these images were called the inverted images. The erected and inverted images for Silk lace are shown in Figures 4(a) and 4(b), respectively. Additionally, to explore the effect of visual field size observation of fabric drape, four window sizes, drastically restricting the visual field by different degrees, were made. The windows for the Satin organdy sample are shown in Figures 5(a)-(d). The three window sizes were $2 \mathrm{~cm} \times 2 \mathrm{~cm}, 4 \mathrm{~cm} \times 4 \mathrm{~cm}$, and $8.5 \mathrm{~cm} \times 8.5 \mathrm{~cm}$, that is, the maximum fabric area that does not include background. These three sizes were called $2 \times 2,4 \times 4$, and $8.5 \times$ 8.5 , respectively. The fourth window size was the no-window image, called N-win. Three hundred and fifty-two images (11 fabrics $\times 4$ drape complexity levels $\times 4$ window sizes $\times 2$ groups) were employed as the experimental stimuli.

\subsubsection{Experimental Procedure, Booth, and Equipment} The experimental procedure is shown in Figure 6.

An observer entered the experimental booth (Figure 7). Ambient light was provided with a fluorescent light fixture in the ceiling of the room. Horizontal and vertical illuminance levels near the center of the display were approximately $523 \mathrm{~lx}$ and $230 \mathrm{~lx}$, respectively. The observer was asked to select the test cloth that matched the image presented on a display by touching 11 test 


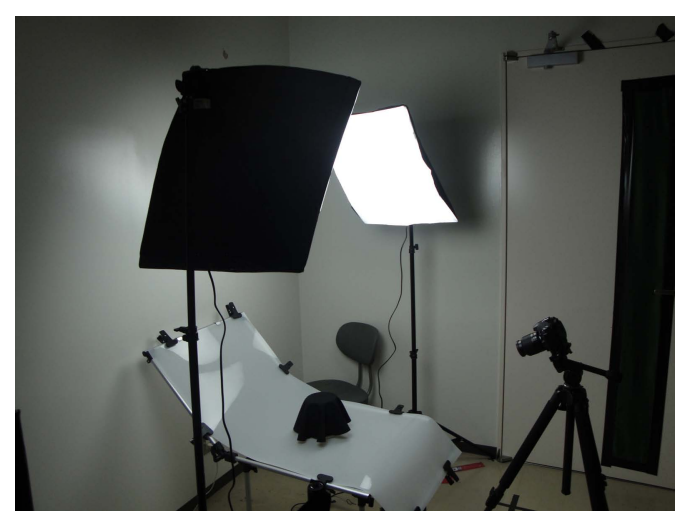

Figure 2: Setup of photography equipment

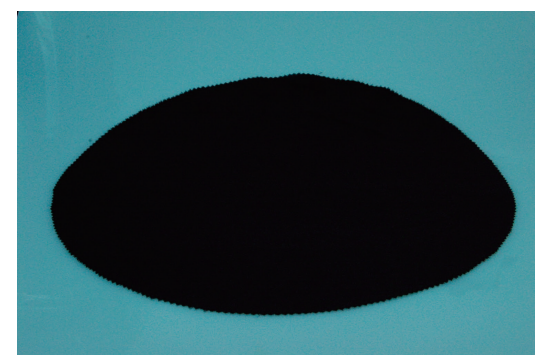

(a) Comp. 0 (drape complexity level 0)

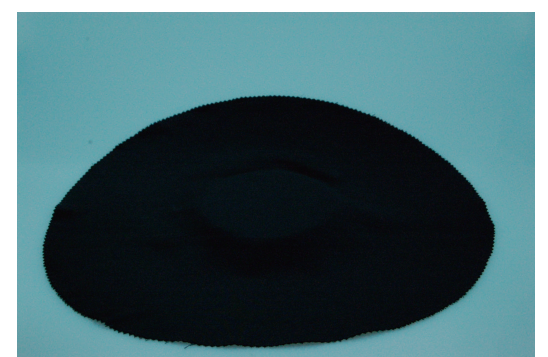

(b) Comp. 1 (drape complexity level 1)

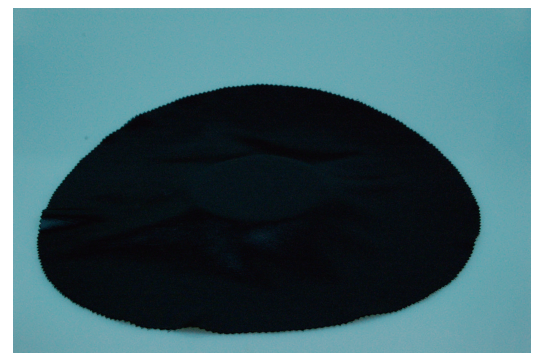

(c) Comp. 2 (drape complexity level 2)

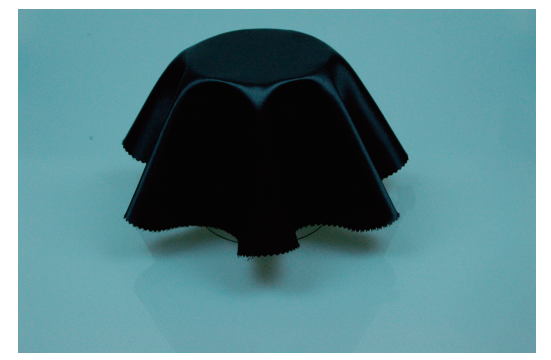

(d) Comp. 15 (drape complexity level 15)

Figure 3: Different levels of drape complexity for Back satin shantung

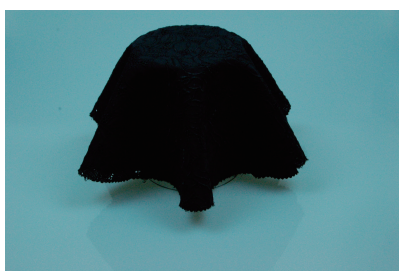

(a) Erected image

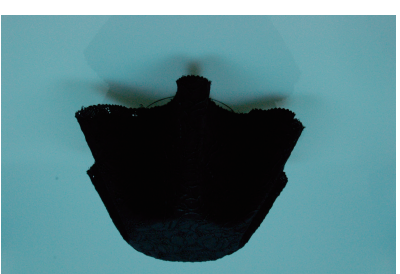

(b) Inverted image
Figure 4: Erected and inverted images for Silk lace

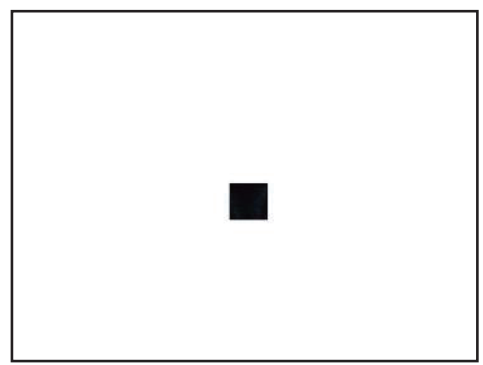

(a) $2 \times 2$

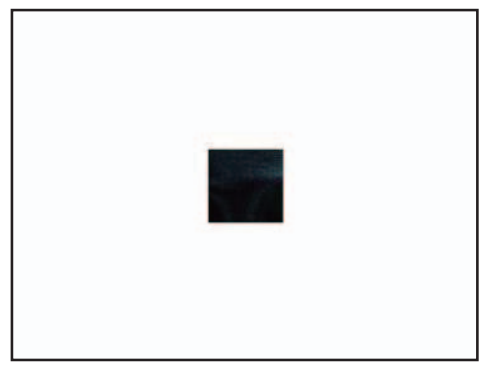

(b) $4 \times 4$

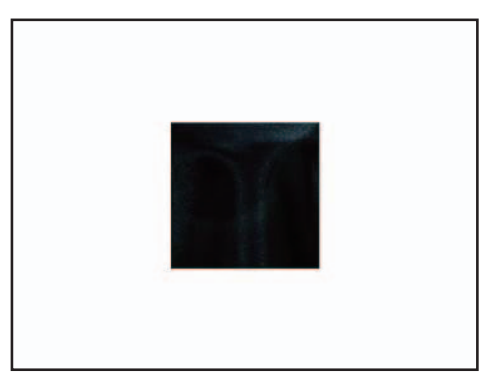

(c) $8.5 \times 8.5$

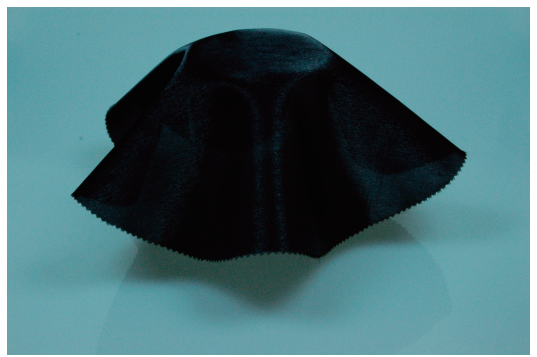

(d) N-win

Figure 5: Experimental stimuli with different window sizes for Satin organdy 


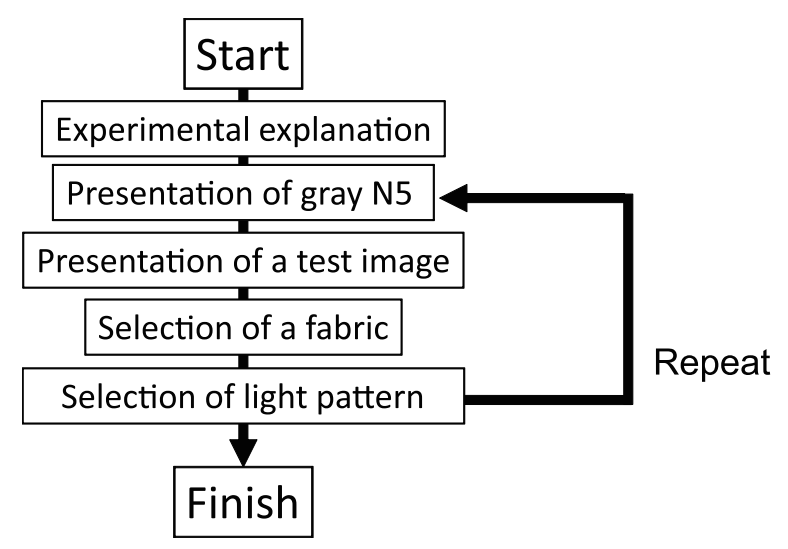

Figure 6: Experimental procedure

cloths without seeing them. The 15.4-in display of a personal computer (VAIO PCG-9S2N) was used to present the stimuli. The viewing distance between the display and the observer's eye was about $60 \mathrm{~cm}$. Each observer was given as much time as he/she needed to carry out the evaluation. To avoid comparison of successive images, a homogeneous gray plane (N5) was presented between each pair of experimental stimuli. Each observer evaluated 352 images in 4 sessions. The order in which experimental stimuli were shown during the sessions was randomized among the subjects. The observers were six engineering students with normal color vision.

To accurately estimate light-source positions, each observer was asked to choose one light pattern from the six possible light patterns presented (shown in Figure 8). The No. 2 and No. 3 light patterns correspond to the erected images and inverted images, respectively.

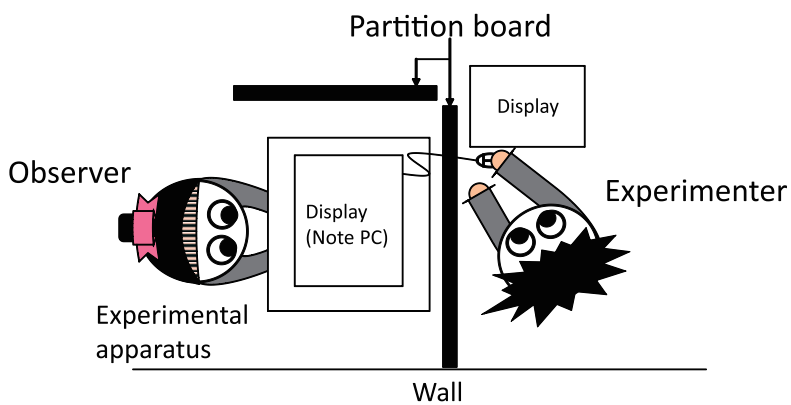

Figure 7: Experimental booth
(1)

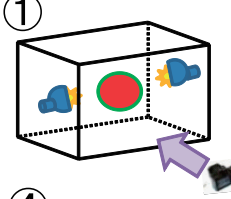

(4)

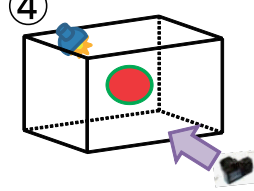

(2)

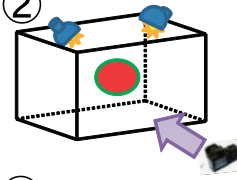

(5)

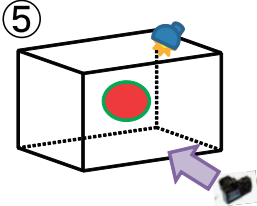

(3)

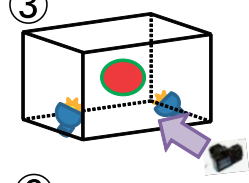

(6)

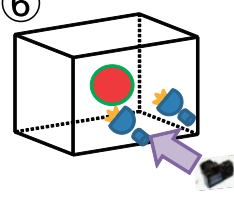

Figure 8: Light patterns

\section{RESULTS AND DISCUSSION}

The number of correct answers for each test cloth in all conditions was counted. The correct answers relative to window sizes, drape complexity levels, and selected light patterns were compared.

Figure 9 shows the total number of correct answers for all test cloths for all combinations of window sizes and drape complexity levels examined here. As shown in the figure, the total number of correct answers increases as the window size changes from $2 \times 2$ to $\mathrm{N}$-win. Two statistically significant differences are observed between $\mathrm{N}$-win and the other window sizes $-2 \times 2,4 \times 4$, and 8.5 $\times 8.5$ - and between $2 \times 2$ and the other window sizes- 4 $\times 4,8.5 \times 8.5$, and $\mathrm{N}$-win. It is assumed that the size of the window has the potential to change the observers' ability to judge the fabric's surface and other properties. On the other hand, the total number of correct answers does not change as the drape complexity level increases. However, in the case of $\mathrm{N}$-win, the total number of correct answers increases only incrementally as the drape complexity level increases (Figure 10). These results

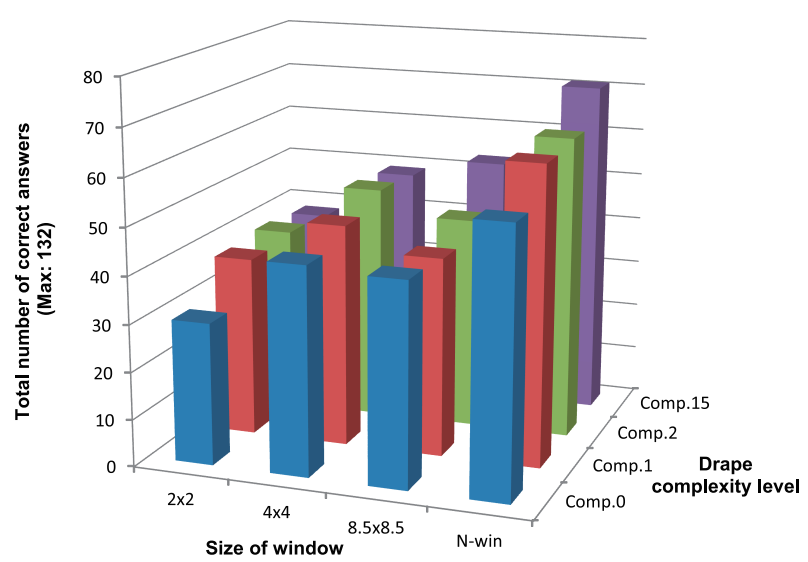

Figure 9: Total number of correct answers

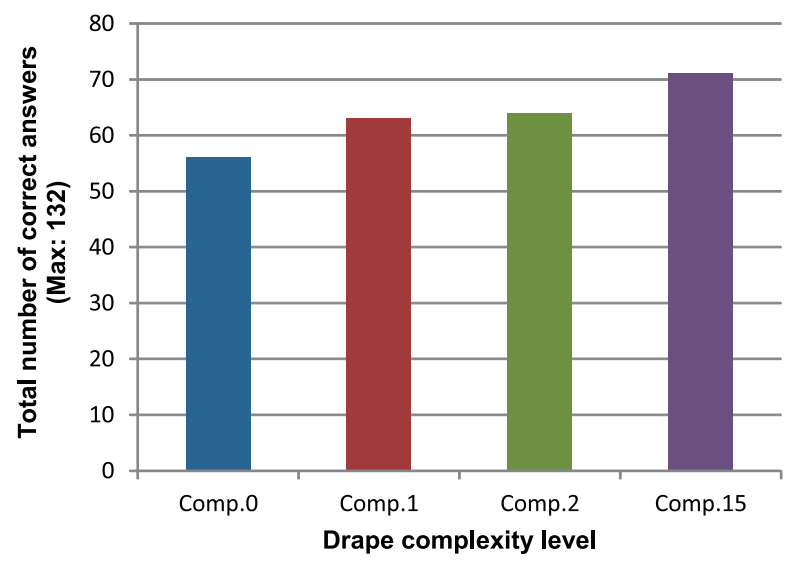

Figure 10: Total number of correct answers in the case of $\mathrm{N}$-win for each drape complexity level 
suggest that when the effect of the window size is removed, the drape complexity level affects the number of correct answers, but the effect of the drape complexity level on cloth texture recognition is weaker than that of the window size.

The number of correct answers for light pattern recognition for all combinations of window size and drape complexity levels is shown in Figure 11. A correct answer included selecting the No. 2 pattern when observing the erected image or the No. 3 pattern when observing the inverted image. The number of correct answers was especially large for Comp.15, the highest drape complexity level. The ratio of correct answers given when observing the erected image to those given when observing the inverted image for Comp.15 is shown in Figure 12. The number of observers who correctly chose the light pattern when observing the erected image increased incrementally with the change in window size. This result suggests that there is a direct relationship between accurately estimating the light positions when observing the erected image for Comp.15 and accurately identifying the fabric in the fabric-identification experi-

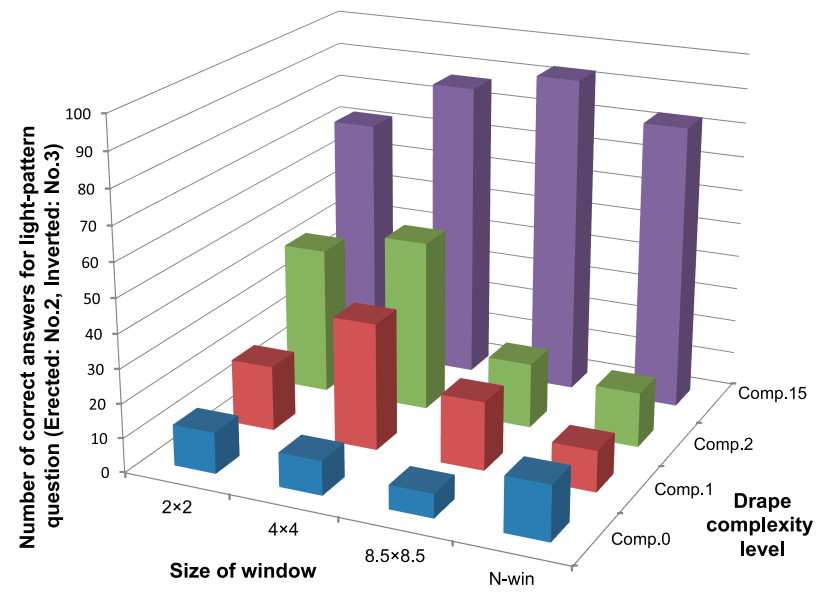

Figure 11: Number of correct answers for light-pattern question

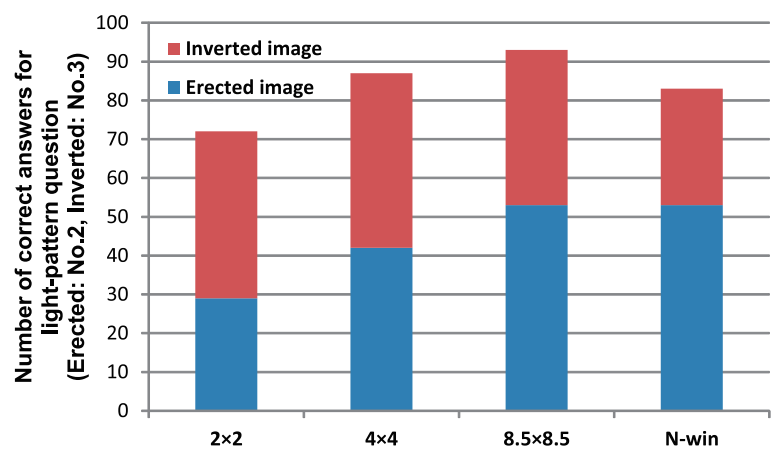

Figure 12: Ratio of correct answers to the light-pattern question when observing the erected image to correct answers when observing the inverted image for Comp. 15 ment. We presume that estimating the light patterns corresponding to the erected image was easier than it was for the inverted image because the No. 2 light pattern is similar to the effect of natural light coming from above; this enhanced estimation ability was used to identify the cloth that correctly matched the test image in the experiment.

\section{CONCLUSION}

To investigate the effect of key visual factors for cloth texture recognition on a person's ability to identify fabric using visual and tactile information, we conducted an experiment focusing on the complexity of fabric drape shape, the size of the window for observing its drape, and the observer's ability to accurately estimate the lightsource position.

Upon comparing the effects of different window sizes, we found that the total number of correct answers increased incrementally with the drastic change in window size from $2 \times 2$ to $\mathrm{N}$-win. Although the four levels of drape complexity elicited roughly the same total number of correct answers, the total number of correct answers in the $\mathrm{N}$-win case increased incrementally as the drape complexity level increased. These results suggest that when the effect of window size is removed, the drape complexity level affects the observer's ability to correctly identify a fabric. However, the effect of the drape complexity level on cloth texture recognition is weaker than that of the drastic changes in window size.

The number of correct answers when estimating light patterns for Comp.15 was especially large, and in the case of observing the erected image, correct answers increased incrementally with the change in window size. This result suggests that there is a direct relationship between accurately estimating light positions when observing the erected image for Comp. 15 and correctly identifying the fabric. We presume that observers could more easily estimate light patterns corresponding to erected images showing fabric drape because the No. 2 light pattern is very similar to natural light coming from above. This enhanced estimative ability was used when identifying the cloth that correctly matched the test image in the experiment.

\section{ACKNOWLEDGEMENTS}

This research was supported by a Grant-in-Aid from the Japan Society for the Promotion of Science (No.22500184, No.24220012 and No.25330316) and the Eminent Research Selected at Utsunomiya University in 2011. 


\section{REFERENCES}

1. http://www.soumu.go.jp/johotsusintokei/whitepaper/ $\mathrm{ja} / \mathrm{h} 23 / \mathrm{index} \cdot \mathrm{html}$

2. http://www.lnet.co.jp/.

3. Eisuke Nakasu; Highly Realistic Communication Technology: Super Hi-Vision, Journal of the Institute of Image Information and Television Engineers, Vol.65, No.9, pp.1276-1281 (2011) (in Japanese).

4. Tomoharu Ishikawa, Kou Sato, Yoshifumi Matsumoto, Kazuya Sasaki, Hiroko Shimizu, and Miyoshi Ayama; Fundamental Study on Texture Recognition of Cloth Image and Material: Comparison between Engineering and Clothing, Journal of Kansei Engineering, Vol.9, No.4, pp.663-674 (2011) (in Japanese).

5. Tomoharu Ishikawa, Kou Sato, Yoshifumi Matsumoto, Kazuya Sasaki, Hiroko Shimizu, and Miyoshi Ayama; Effect of Knowledge and Experience of Fabric on the Cross-Modal Linkage between Tactile Sensation and Visual Image of Solid Black and Multi-Color Printed Fabrics, Midterm Meeting of the International Colour Association (AIC) 2011 (2011).

6. Enrique Cerda, Lakshminarayanan Mahadevan, and José Miguel Pasini; The Elements of Draping, Proceedings of the National Academy of Sciences (PNAS), Vol.101, No.7, pp.1806-1810 (2004).

7. Shinya Saida and Mitsuo Ikeda; Useful Visual Field Size for Pattern Perception, Perception \& Psychophysics, Vol.25, No.2, pp.119-125 (1979).

8. Chie Muraki Asano, Takako Fujimoto, Akira Asano, Machiko Murakami, Steve McNeil, Surinder K. Tandon, Carolyn Piper, Miyuki Morita, and Masukuni Mori; Evaluation of Kansei of Clothing Materials: Part II-Texture and Visual Impressions Using Novel Technique, Proceedings of the International Conference on Kansei Engineering and Emotion Research 2007, F-6 (2007).

9. JIS L 1096 (1999).

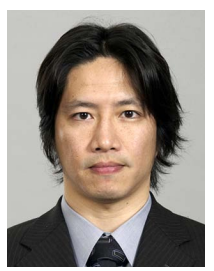

\section{Tomoharu ISHIKAWA}

Tomoharu Ishikawa is an Associate Professor of the Department of Advanced Interdisciplinary Sciences, Graduate School of Engineering, Utsunomiya University, Japan, since 2012. He received the Ph.D. degree in information science from Japan Advanced Institute of Science and Technology in 2001. Since 2001, he has been working for a Research Associate of Japan Society for the Promotion of Science, and an Assistant of Japan Advanced Institute of Science and Technology, and then an Assistant Professor of the Department of Advanced Interdisciplinary Sciences, Graduate School of Engineering, Utsunomiya University. He has been engaged in multisensory psychophysics, psychophysiology and KANSEI Engineering etc. He is now councilor of Japan Society of KANSEI Engineering.

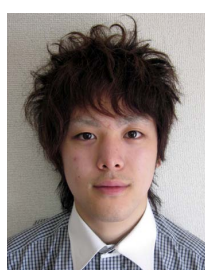

\section{Kou SATO}

Kou Sato is a student of the Department of Advanced Interdisciplinary Sciences, Graduate School of Engineering, Utsunomiya University, Japan, since 2011. And, He performs the study on relationship between visual and tactile perception for cloth texture recognition.

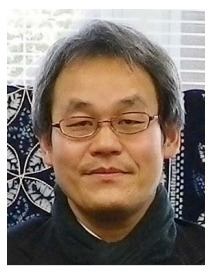

\section{Kazuya SASAKI}

Kazuya Sasaki is an Associate Professor of the Faculty of Education, Utsunomiya University, Japan, since 2001. He received the $\mathrm{Ph}$. D. degree in textile science from Shinshu University, Japan, in 1998. He has been engaged in textile science, especially, measuring the relation between human and clothing, and KANSEI Engineering. Recently, he researches the environmental education based on making things "MONODUKURI" by traditional dyeing and weaving from the viewpoint of KANSEI Education. He is now councilor of Japan Society of KANSEI Engineering.

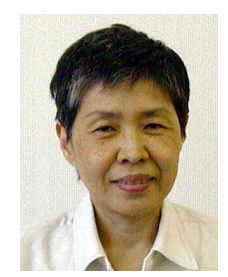

\section{Hiroko SHIMIZU}

Hiroko Shimizu is a Professor of the Faculty of Education, Utsunomiya University, Japan, since 1981. She received the Ph.D. degree from Oosaka City University in 1993. She has been engaged in Textile Science, KANSEI Engineering, and KANSEI Education. She is now councilor of Japan Society of KANSEI Engineering.

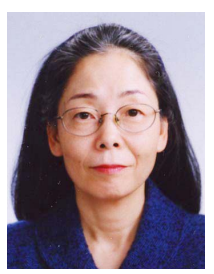

\section{Miyoshi AYAMA}

Miyoshi Ayama is a Professor of the Department of Advanced Interdisciplinary Sciences, Graduate School of Engineering, Utsunomiya University, Japan, since 2008, after having contributed for 15 years as a Professor of Department of Information Science at Utsunomiya University, Japan. She has been engaged in visual psychophysics, color science, and KANSEI Engineering. She is now an associate director of the Center for Optical Research and Education at Utsunomiya University, also an associate director of the CIE (Commission Internationale de l'Éclairage) Division 1, Vision and Color, and a board member of Japanese CIE. 